\title{
GENERAL ALGEBRAIC SOLUTIONS IN THE LOGIC OF CLASSES.
}

BY PROFHSSOR L. M. HOSKINS.

(Read before the San Francisco Section of the American Mathematical Society, February 29, 1908.)

THE following treatment of the problem of inference in the logic of classes possesses some interest from its analogy to general solutions in ordinary algebra. The character of the general solutions here considered is most simply illustrated by what may be called the generalized problem of the syllogism, which may be stated as follows:

Let $x, y, z$ be three class symbols, and let

$$
f_{1}(x, y)=0, \quad f_{2}(y, z)=0,
$$

be any two propositions involving $x, y$ and $y, z$ respectively; then it is required to deduce a proposition

$$
f_{3}(x, z)=0
$$

involving $x$ and $z$ but not $y$.

The most general forms of the above propositions are (writing $x^{\prime}$ for $1-x$, etc.)

$$
\begin{aligned}
& f_{1}(x, y)=l_{1} x y+l_{2} x y^{\prime}+l_{3} x^{\prime} y+l_{4} x^{\prime} y^{\prime}=0, \\
& f_{2}(y, z)=m_{1} y z+m_{2} y z^{\prime}+m_{3} y^{\prime} z+m_{4} y^{\prime} z^{\prime}=0, \\
& f_{3}(x, z)=n_{1} x z+n_{2} x z^{\prime}+n_{3} x^{\prime} z+n_{4} x^{\prime} z^{\prime}=0,
\end{aligned}
$$

in which $l, m, n$ are numerical coefficients ; and the non-vanishing of any coefficient (as $m_{2}$ ) implies the vanishing of the corresponding class term $\left(y z^{\prime}\right)$. The problem is to express the coefficients in (3) in terms of those in (1) and (2).

A solution is obtained in simple and symmetrical form by regarding (1), (2), and (3) as particular cases of the most general proposition involving $x, y$, $z$,

(4) $f(x, y, z)=a x y z+b x y z^{\prime}+c x y^{\prime} z+d x y^{\prime} z^{\prime}+e x^{\prime} y z+$

By Boole's rule of elimination

$$
f x^{\prime} y z^{\prime}+g x^{\prime} y^{\prime} z+h x^{\prime} y^{\prime} z^{\prime}=0 \text {. }
$$

$$
f_{1}(x, y)=f(x, y, 1) f(x, y, 0) .
$$


Hence (1), (2), and (3) may be written

$$
\begin{aligned}
& a b x y+c d x y^{\prime}+e f x^{\prime} y+g h x^{\prime} y^{\prime}=0, \\
& a e y z+b f y z^{\prime}+c g y^{\prime} z+d h y^{\prime} z^{\prime}=0, \\
& a c x z+b d x z^{\prime}+e g x^{\prime} z+f h x^{\prime} z^{\prime}=0 .
\end{aligned}
$$

It is now easy by inspection to determine whether the nonvanishing of particular coefficients in (5) and (6) implies the non-vanishing of any coefficients in (7). For example, the non-vanishing of $a b$ and $c g$ implies the non-vanishing of $a c$; $i$. e., the premises

$$
x y=0, \quad y^{\prime} z=0
$$

imply the conclusion $x z=0$. On the other hand, no conclusion can be drawn from

$$
x y=0, \quad y z^{\prime}=0
$$

since the non-vanishing of $a b$ and $b f$ does not require the nonvanishing of any coefficient in (7).

The above refers primarily to so-called universal propositions ; but the solution includes also particular propositions, if these are understood as affirming the existence of the classes referred to. Thus in (5), if any coefficient, as $c d$, is made zero while all others remain arbitrary, the proposition affirms the existence of the class $x y^{\prime}$, since the four classes $x y, x y^{\prime}, x^{\prime} y, x^{\prime} y^{\prime}$ cannot all be assumed to vanish without denying the existence of the " universe of discourse." As an example of inference when one premise is particular, notice that if $c d$ vanishes, while $c g$ does not, we must have $d=0$ and therefore $b d=0 ; i$.e., from the premises

$$
x y^{\prime} \neq 0, \quad y^{\prime} z=0,
$$

may be inferred the conclusion $x z^{\prime} \neq 0$.

Propositions involving more than three primary class terms may be treated in like manner, but the application to particular cases becomes rapidly less simple as the number of primary classes increases.

The method applies also to the case in which $x, y, z$ represent propositions instead of classes; but interpretations in the logic of propositions involve some peculiar subtleties. 